\title{
Review of active textile antenna co-design and optimization strategies
}

\author{
Arnaut Dierck \\ Department of Information Technology \\ Ghent University \\ Ghent, Belgium \\ Email: arnaut.dierck@intec.ugent.be
}

\author{
Frederick Declercq \\ Department of Information Technology \\ Ghent University \\ Ghent, Belgium
}

\author{
Hendrik Rogier \\ Department of Information Technology \\ Ghent University \\ Ghent, Belgium
}

\begin{abstract}
This paper describes the challenges that arise in active wearable textile antenna design and optimization. After a short introduction, design strategies for two cases with different needs are discussed and examples are given for each design strategy. In the first case, a low-noise amplifier is connected directly to a $2.45 \mathrm{GHz}$ ISM-band antenna by optimizing the antenna impedance to match the low-noise amplifier input impedance for optimal noise performance. In the second case, an aperture-coupled GPS antenna incorporating a discrete $50 \Omega$ hybrid coupler is linked to a low-noise amplifier by means of a matching network to match the $50 \Omega$ hybrid coupler port to the low-noise amplifier impedance for optimal noise performance.
\end{abstract}

\section{INTRODUCTION}

In the last decades we witnessed a rapid growth in the field of ubiquitous computing, which not only encompasses a growing amount of ever smaller electronic devices but even more so the interaction between them. Recently, the field of smart textiles, where electronic systems are unobtrusively integrated into garments, has received a great amount of attention. Such textiles offer new functionalities to our future garments, enhancing the quality of life without sacrificing the comfort of the wearer. This emerging technology finds its use in applications ranging from sportswear to healthcare, public service and military apparel. Given its size, the wearable antenna forms a vital component in any such a system. This has led to a wide range of wearable antennas for on- and off-body communications being reported in literature [1]-[9]. Even though transmit power levels are limited for personal area networks due to vicinity of the human body, the wearable antennas must provide highly reliable communication links, especially in emergency situations and for healthcare applications. The proximity of the human body not only limits permitted transmit power levels, but also significantly affects antenna performance. For example, higher losses due to bulk power absorption, radiation pattern distortion, shift in resonance frequency and changes in antenna input impedance due to capacitive coupling with the body have been observed [10], [11]. Antennas on EBG substrates were reported to overcome radiation absorption by the body and achieve higher antenna gain [12], [13]. Other effects resulting from the integration of antennas in garments have also been studied, such as for example antenna bending [14], [15] and changing substrate moisture levels [16]. As a means to improve the reliability of the communication links, active antennas are a logical step up from designing the passive antenna and active transceiver separately. Active antennas allow for more compact and more efficient designs by integrating active electronic circuitry together with the passive radiator structure. This also benefits system ruggedness thanks to omitting potentially weak cable links between body-worn antennas and transceivers. In this contribution, active antennas consisting of a radiating element and a low-noise amplifier (LNA) will be discussed. Depending on particular constraints, different design strategies can be chosen. Two different cases and their corresponding design strategies will be discussed and illustrated. In the first case, the design of a wearable active receiving antenna for communication in the $2.45 \mathrm{GHz}$ ISM band is addressed [17]. This antenna is designed for an optimal noise match impedance with its terminals directly coupled to the LNA input. The second case deals with an active wearable GPS receiver antenna [18]. This design involves an antenna element that is circularly polarized by means of an off-the-shelf $50 \Omega$ discrete hybrid coupler, enforcing $50 \Omega$ terminations at the ports of the radiating element and the LNA, so use of a matching network is needed.

\section{Case I: Design of a Wearable $2.45 \mathrm{GHz}$ Active RECTANGULAR RING ANTENNA}

As a first case, the design of a wearable active antenna for communication in the $2.4-2.485 \mathrm{GHz}$ ISM band is discussed. First, the topology of the antenna and LNA is described. Next, the chosen design strategy is elaborated. Finally, the design strategy is evaluated by means of measurements.

\section{A. Active Antenna Topology}

The topology of the active receiving antenna with integrated low-noise amplifier directly located underneath the ground plane is depicted in Fig. 1. The radiating element for the 2.4-2.485 GHz ISM band is a rectangular ring antenna [19]. To avoid matching network losses degrading the system noise performance, the antenna signal is directly fed into the LNA through a via without a matching network of any kind. Long microstrip lines were avoided as well, to minimize losses that might occur due to use of electrotextiles as conductors and non-conductive textiles as substrates. The wearability of 


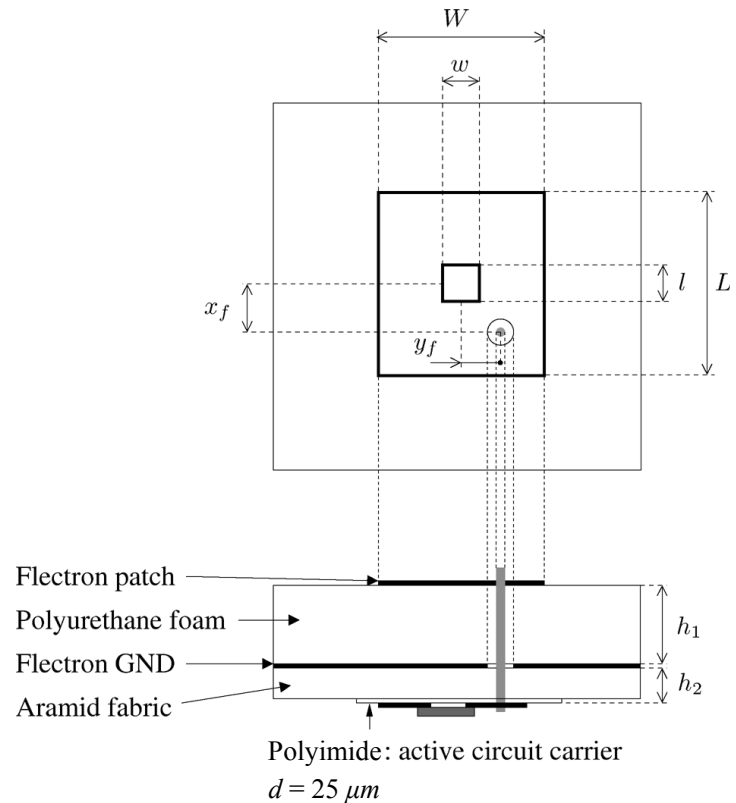

Fig. 1. Wearable active rectangular ring antenna topology

TABLE I

Electromagnetic Properties of the Active Antenna Materials

\begin{tabular}{c|c|c|c} 
& $\epsilon_{r}$ & $\tan \delta$ & materials \\
\hline $\begin{array}{c}\text { Antenna substrate } \\
h_{1}=3.56 \mathrm{~mm}\end{array}$ & 1.28 & 0.016 & polyurethane foam \\
\hline $\begin{array}{c}\text { LNA substrate } \\
h_{2}=425 \mu \mathrm{m}\end{array}$ & 1.84 & 0.015 & $\begin{array}{c}\text { aramid textile fabric } \\
+ \text { polyimide layer }\end{array}$ \\
\hline \hline & $\begin{array}{c}R_{s} @ 2.45 \mathrm{GHz} \\
{[\Omega / \mathrm{sq}]}\end{array}$ & $\sigma[\mathrm{S} / \mathrm{m}]$ & \\
\hline Flectron & 0.45 & $4.8 \times 10^{4}$ &
\end{tabular}

the active antenna is ensured by using mainly breathable textiles for the antenna as well as for the LNA substrate. The small active circuit including the LNA interconnections is implemented on a thin flexible copper-on-polyimide film. The different substrate layers and their characteristics are listed in Table I. The electromagnetic properties of the materials were extracted by means of the techniques presented in [20]. The substrate and conductive layers were glued together by means of an adhesive sheet. The via connecting the radiating patch to the LNA and the vias of the LNA circuit consist of $1 \mathrm{~mm}$ and $0.5 \mathrm{~mm}$ diameter copper wire, respectively. The LNA was built around an AVAGO ATF-54143 pHEMT transistor using a grounded-source topology [21]. The schematic is shown in Fig. 2. The transistor bias $\left(V_{d s}=3 \mathrm{~V}, I_{d}=60 \mathrm{~mA}\right.$, and $V_{g s}$ $=0.56 \mathrm{~V})$ is accomplished by the resistive voltage divider $R_{1}-R_{2}-R_{3}$. The gate bias is delivered through inductor $L_{1}$, whereas the drain bias is provided over the $\lambda / 4$ transmission line. Resistive damping by means of $R_{5}$ and the bypass capacitor $C_{2}$ improves low-frequency stability without degrading the LNA performance. Resistive loading of the drain with $R_{6}$ provides additional stability. $C_{3}$ is used as a decoupling capacitor, whereas $L_{2}$ is used for good output matching.

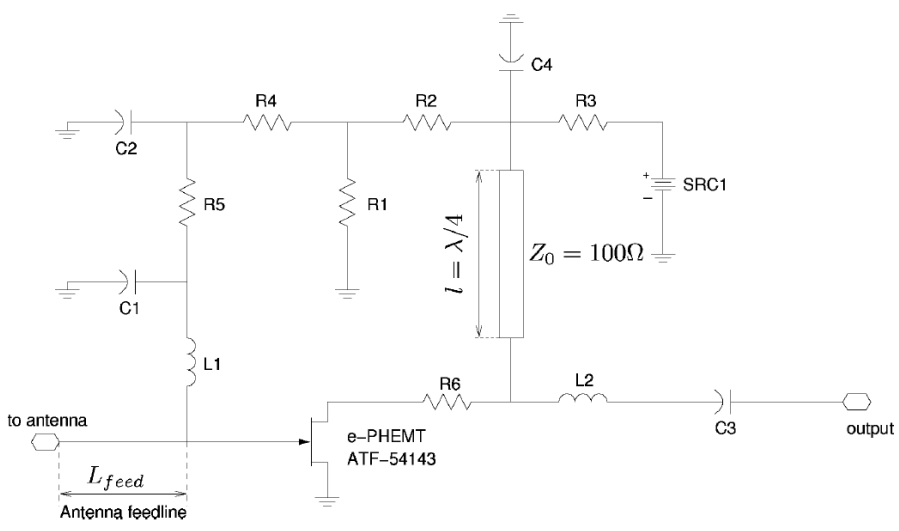

Fig. 2. Schematic of the LNA

\section{B. Active Antenna Design Strategy}

Because of the direct integration of the patch antenna and the LNA into a compact design, a joint circuit/full-wave optimization is required to simultaneously design the antenna and LNA to meet the desired specifications. The design flow is sketched in Fig. 3. The gist of the design strategy is to control the complex antenna impedance and match it to the impedance for optimal noise performance of the LNA. Therefore, the antenna dimensions and several well-chosen parameters of the LNA were chosen as degrees of freedom for the optimization cycles. To account for the losses induced by the Flectron ground plane, a multi-platform design approach is required since a finite conductivity cannot be defined for a ground plane embedded between two substrates in ADS Momentum. Starting in ADS, the active circuitry was completely modeled on the LNA substrate as defined in Table I and this without the via connecting the antenna to the LNA, allowing the inand output port of the LNA to be in the same plane as the passive interconnections in ADS Momentum. In this way it was possible to define an infinite ground plane with a bulk conductivity of $4.8 \times 10^{4} \mathrm{~S} / \mathrm{m}$. A co-simulation was set up for the LNA design, modeling the passive interconnections in the ADS Momentum full-wave simulator and the lumped passive and active components in the ADS circuit simulator by means of scattering parameter libraries to account for parasitic effects at high frequencies. The passive antenna radiator was modelled in the full-wave frequency domain simulator of CST Microwave Studio, where the finite Flectron ground plane and patch and the substrates were defined according to the values in Table I. The computational cost was limited by adopting the following design strategy. First, an LNA topology exhibiting a low noise figure and good linearity was selected. Co-optimization of the LNA interconnections and the lumped components yielded the required antenna impedance $Z_{\text {opt }}=$ $35.015-17.644 j \Omega$, which resulted in a minimum achievable noise figure $N F_{\text {min }}=0.715 \mathrm{~dB}$ and a noise-matched power gain of $12.939 \mathrm{~dB}$. Unconditional stability of the design was ensured using the stability factors $K$ and $B_{1}$ [22].

Second, starting from a $50 \Omega$ matched design in the $2.45 \mathrm{GHz}$ ISM band, the feed positions $x_{f}$ and $y_{f}$ of the 


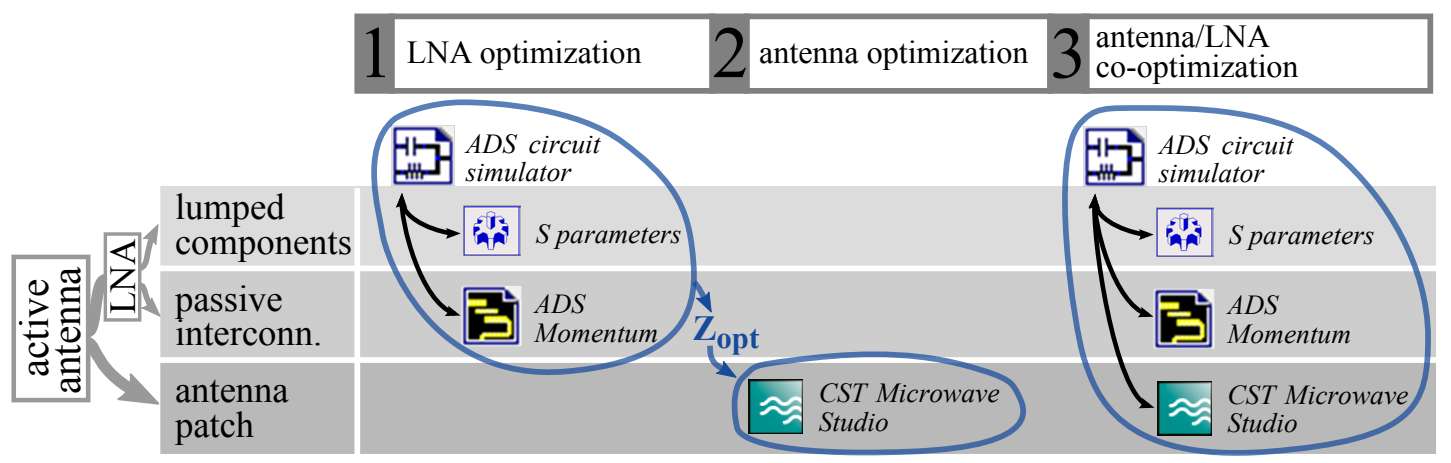

Fig. 3. Design flow of the active receiving antenna

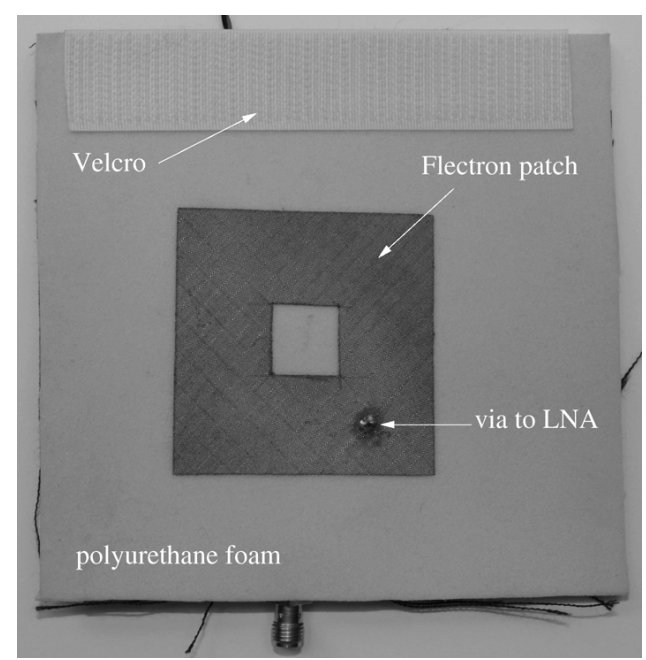

Fig. 4. Picture of the patch antenna of the active receiving antenna

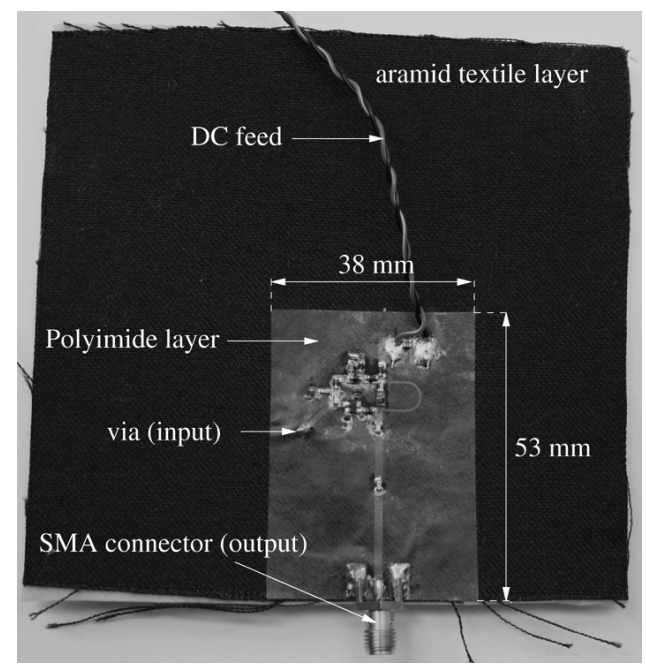

Fig. 5. Picture of the LNA of the active receiving antenna

rectangular ring antenna were optimized by means of a random search method in the full-wave frequency domain solver of CST to minimize the error function $\left|\Gamma_{g}-S_{o p t}\right|$ at $2.45 \mathrm{GHz}, \Gamma_{g}$ being the antenna reflection coefficient and $S_{\text {opt }}$ the optimal reflection coefficient for minimum LNA noise figure (corresponding to $Z_{\text {opt }}=35.015-17.644 j \Omega$ ). In a final EM/circuit co-design step, the complex antenna impedance and the LNA S-parameters were interfaced in a dynamic link between CST Microwave Studio and ADS Momentum coordinated from within the ADS circuit simulator, optimizing the antenna parameters $L, W, l_{g a p}, w_{g a p}, x_{f}$ and $y_{f}$ together with the LNA parameters $L_{1}, L_{2}$ and $L_{\text {feed }}$ to meet the specifications. To this aim, the following design goals were adopted in the entire ISM band: an LNA noise figure equal to the minimum achievable noise figure $N F_{\text {min }}$, sufficient available gain $G_{A}$, a flat gain response $\left(G_{A, \max }-G_{A, \min }<0.5 \mathrm{~dB}\right)$ and output matching $\left|\Gamma_{\text {out }}\right|<-10 \mathrm{~dB}$. Due to the many degrees of freedom in the optimization process, multiple solutions will meet the requirements. Simulated annealing was used to find the global maximum. The optimized design was subsequently manufactured, resulting in an active antenna measuring $11 \mathrm{~cm}$ $\times 11 \mathrm{~cm}$. Breathability of the design was safeguarded by limiting the dimensions of the polyimide layer while ensuring a good adhesion between the aramid and polyimide layers. The front- and backside of the fabricated wearable active antenna are shown in Figs. 4 and 5, respectively.

\section{Design Evaluation}

To evaluate the design strategy, simulated active antenna performance is compared with measurements performed in an anechoic chamber. First, the LNA performance is measured seperately, both on- and off-body. Next, the active antenna performance is examinated. For the on-body measurements, the LNA/active antenna is integrated between the inner liner and the thermal/moisture barrier of a fire fighter jacket, as depicted in Fig. 6.

1) LNA Performance Validation: The LNA was measured in a $50 \Omega$ environment by connecting a through-hole SMA connector at its input. The Agilent Technologies N5241A PNA-X Vector Network Analyzer was used to perform twoport scattering parameter measurements in the $0.1-6 \mathrm{GHz}$ range, as well as noise figure measurements by means of the source-corrected noise measurement technique embedded in the PNA-X measurement system [23]. In Fig. 7 the LNA gain and noise figure are depicted. For the on-body measurement, the LNA was integrated at position 1 inside the garment 


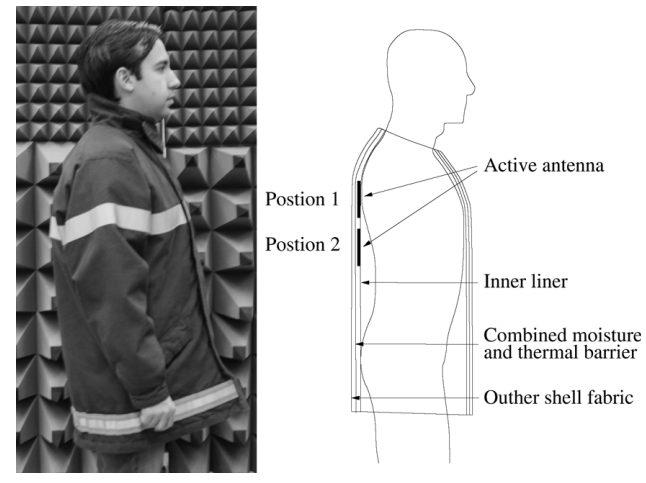

Fig. 6. LNA/antenna positions for the on-body measurements

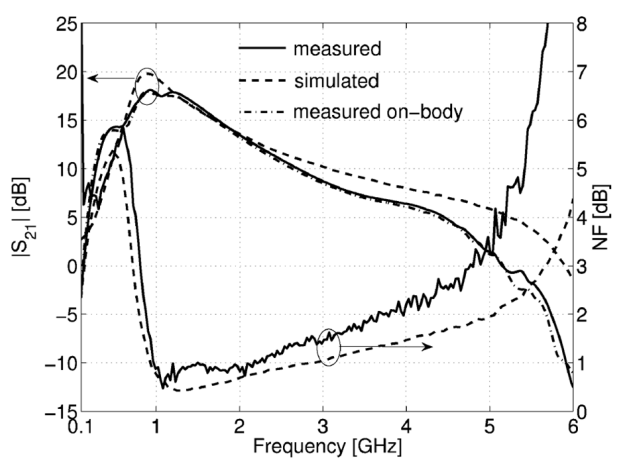

Fig. 7. LNA gain and noise performance

as shown in Fig. 6. A good agreement of the measured and simulated data is observed over the measured frequency range. At $2.45 \mathrm{GHz}$ the simulated $\left|S_{21}\right|$ and $\mathrm{NF}$ are $11.79 \mathrm{~dB}$ and $0.87 \mathrm{~dB}$, respectively, compared to measured values of $11.18 \mathrm{~dB}$ and $1.25 \mathrm{~dB}$. Note that the $\mathrm{S}$-parameter and noise simulations did not include the through-hole SMA connector, as the finite conductivity of the ground plane allowed only a single layer substrate definition in ADS Momentum. The on-body gain measurements show that the LNA power loss is neglegible notwithstanding the unshielded body-directed placement in the garment. The LNA on-body gain is $10.87 \mathrm{~dB}$. Stability factors extracted from the measured S-parameters showed an unconditionally stable LNA.

2) Active Antenna Performance Validation: Due to the integration of the amplifier and antenna in a single entity, characterization of amplifier gain and noise figure can no longer be performed by traditional two-port methods. A measurement technique similar to that in [24] was adopted, where the gain of the amplifier is extracted by comparing the active antenna gain with the gain of an identical passive antenna and the noise figure is determined based on the active antenna's transducer gain and absolute noise power density at the output referred to $290 \mathrm{~K}$. According to the aforementioned procedure, the results depicted in Fig. 8 were obtained for the off-body measurement, which show a good agreement with the simulated active antenna performance. The gain for on-and off body positions is shown in Fig. 9. We notice that power losses for the active and

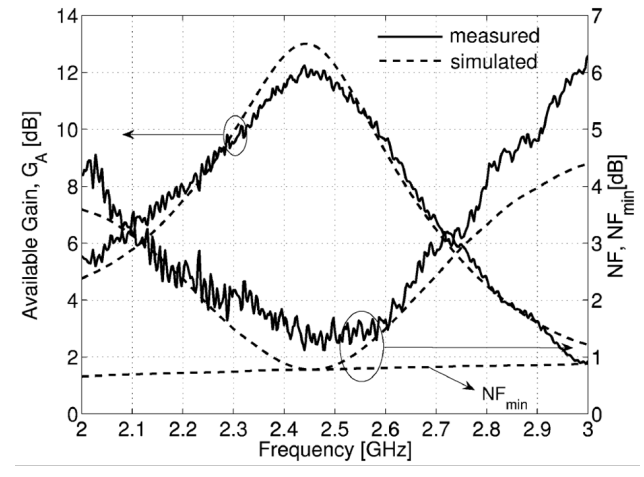

Fig. 8. Active antenna noise figure NF and available gain $G_{A}$

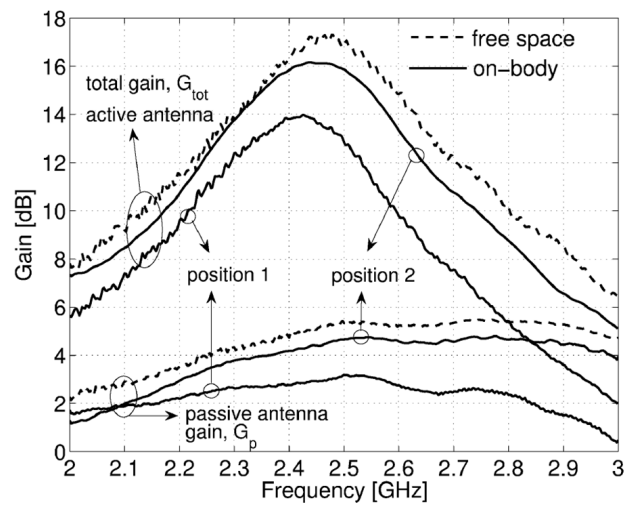

Fig. 9. Active receiving antenna simulated and measured gain

passive antenna at different positions are very comparable. As stated in section II-C1, the LNA power loss due to proximity of the human body is neglegible, attributing the bulk of the active antenna power losses to radiation absorption by the human body. The measured active antenna return loss was lower than $-10 \mathrm{~dB}$ over the complete ISM-band.

\section{Case II: Design of A Wearable Active GPS ANTENNA}

The second case discusses the design of a wearable active GPS antenna. This antenna is a circularly polarized aperturecoupled microstrip patch antenna for use in the GPS L1-band centered at $1.57542 \mathrm{GHz}$. First the topology of the antenna and LNA is described. Afterwards the adopted design strategy is elaborated. Finally the design strategy is evaluated by means of measurement results.

\section{A. Active Antenna Topology}

The geometry of the active aperture-coupled patch antenna is shown in Fig. 10. The LNA is located directly underneath the antenna ground plane. To construct the antenna, flexible materials were used: polyurethane foam for the antenna substrate, aramid fabric for the feed substrate and copperon-polyimide film for the conducting structures. The active antenna layers were glued together by means of a thermally activated adhesive sheet. The characteristics of the wearable substrates are presented in Table II. To realize a right-handed 


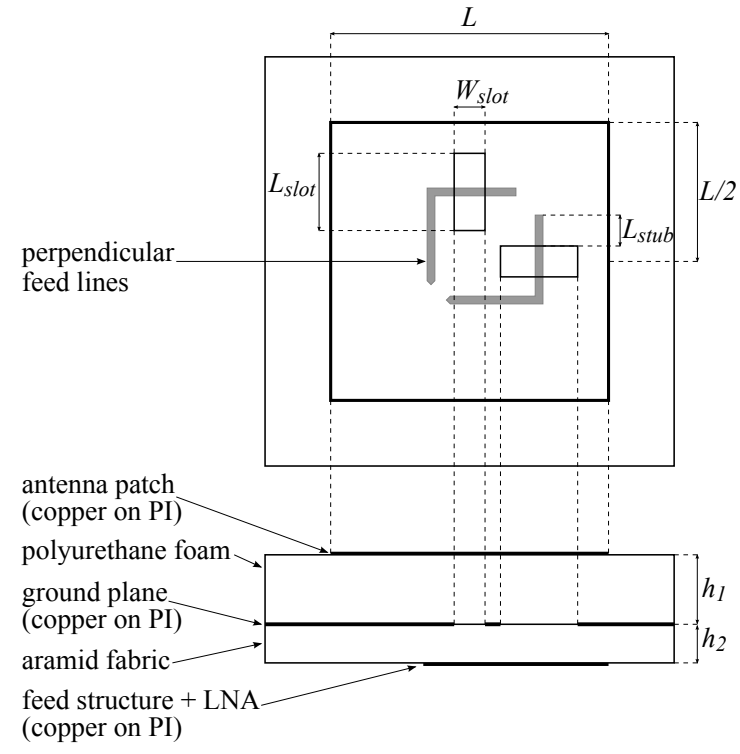

Fig. 10. Wearable active GPS antenna topology

circular polarization, the antenna consists of a square patch excited through two perpendicular rectangular slots in the ground plane. On the feed substrate underneath this ground plane two perpendicular feed lines are routed to a discrete quadrature hybrid coupler. Aperture coupling was chosen to avoid the use of vias through the polyurethane substrate, augmenting the antenna flexibility and robustness. The centered position of the slots allows for a strong excitation of two orthogonal modes on the patch, whereas the quadrature hybrid produces a $90^{\circ}$ phase difference between the modes over a wide frequency range. The realization of this phase difference by means of a discrete component, as opposed to e.g. asymmetries in the patch and/or feed structure ensures its robustness, as the discrete component provides circular polarization over a large frequency band. The quadrature hybrid is connected to the LNA input via a matching network needed to match the LNA optimum noise impedance to the $50 \Omega$ discrete quadrature hybrid. The LNA was built around the AVAGO ATF-54143 pHEMT transistor in a grounded-source setup. The layout of this LNA is shown in Fig. 11. As this circuit bears a lot of resemblance to the one discussed in Case I, we refer to section II-A for the functional description of most components. A transistor bias $V_{d s}=3 \mathrm{~V}$, $I_{d}=24.4 \mathrm{~mA}$ and $V_{g s}=0.46 \mathrm{~V}$ was chosen. Differences with the Case I LNA are the in- and output matching networks, for which capacitor-stub-capacitor filters were used, and the $\lambda / 4$ transmission line $T L_{4}$ connecting the LNA output to the $3 \mathrm{~V}$ DC voltage supply pad. This transmission line in combination with capacitance $C_{10}$ provides a high impedance at the GPS L1 frequency allowing a $3 \mathrm{~V}$ DC voltage to be supplied via the LNA output. This method of supplying a DC voltage is used when connecting the active antenna to a GPS receiver unit with a built-in DC bias [25].
TABLE II

Electromagnetic Properties of the ACtive Antenna Materials

\begin{tabular}{c|c|c|c} 
& $\epsilon_{r}$ & $\tan \delta$ & materials \\
\hline \hline $\begin{array}{c}\text { Antenna substrate } \\
h_{1}=3.56 \mathrm{~mm}\end{array}$ & 1.25 & 0.016 & polyurethane foam \\
\hline $\begin{array}{c}\text { LNA substrate } \\
h_{2}=400 \mu \mathrm{m}\end{array}$ & 1.99 & 0.015 & aramid textile fabric
\end{tabular}

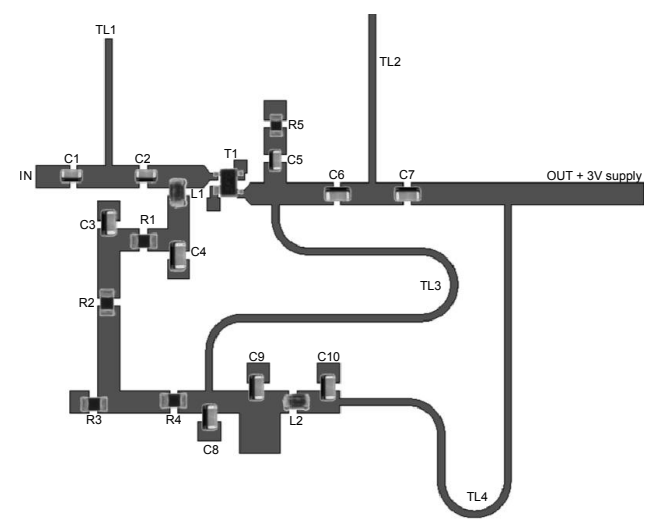

Fig. 11. Layout of the GPS antenna LNA

\section{B. Active Antenna Design Strategy}

As opposed to Case I, we do not have the liberty to adjust the antenna impedance to the optimal noise impedance for the LNA since the discrete quadrature hybrid is a $50 \Omega$ matched component, imposing $50 \Omega$ matching requirements on both the antenna and the LNA. The design flow is outlined in Fig. 12. Perfect electric conductors are used to model the copper conductors in the active antenna, allowing the design to be completely modeled in ADS Momentum. In a first step, the antenna was designed in the ADS Momentum full-wave environment and optimized for optimal return loss $\left|S_{11}\right|$ from within the ADS circuit simulator. Since optimization on the axial ratio cannot be performed automatically in the ADS circuit simulator, parameter sweeps on the stub and slot dimensions were used to finetune the axial ratio. In later designs, a link between Matlab and ADS Momentum was established, enabling a Matlab optimizer to access the axial ratio. In these simulations the discrete quadrature coupler was not included, but a phase difference was imposed in the Momentum postprocessing. Second, for the LNA, a similar simulation setup as in Case I was used, modelling the passive interconnections in the ADS Momentum full-wave environment and the lumped elements in the ADS circuit simulator. The following design specifications were put forward: high gain $\left(\left|S_{21}\right|>15 \mathrm{~dB}\right)$, good in- and output matching $\left(\left|S_{11}\right|\right.$ and $\left|S_{22}\right|<-10 \mathrm{~dB}$ ), noise figure NF around $0.5 \mathrm{~dB}$ and unconditional stability (taken into account by means of stability factors $K$ and $B_{1}$ ). An optimization was performed on the lumped component values and the lengths of the stub waveguides in the inand output matching networks. The stub waveguides were modeled separately from the rest of the LNA layout during the optimization in order to avoid lengthy computation of 


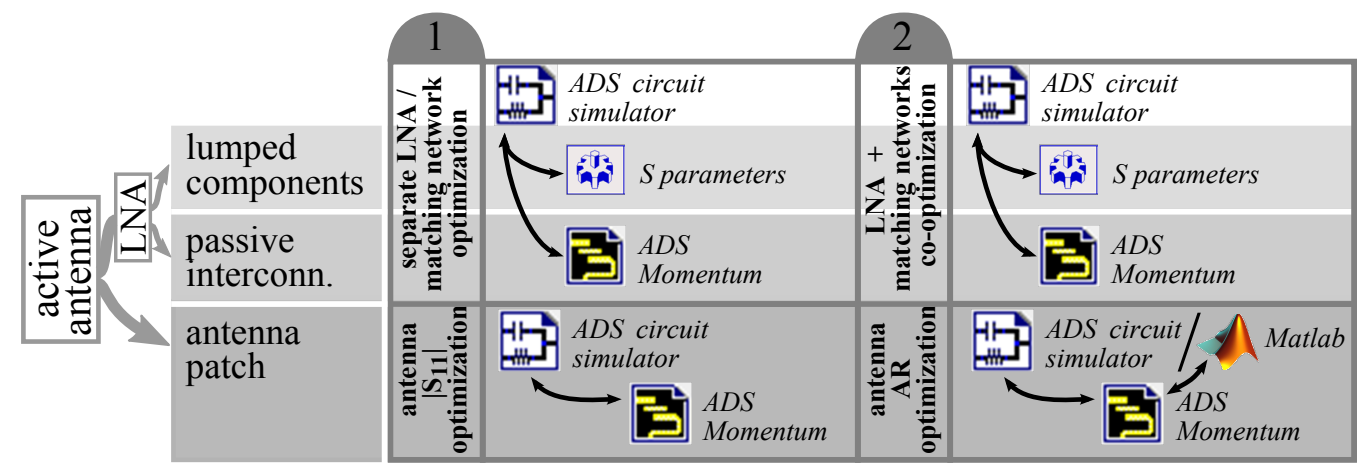

Fig. 12. Parallel design flow of the active GPS antenna

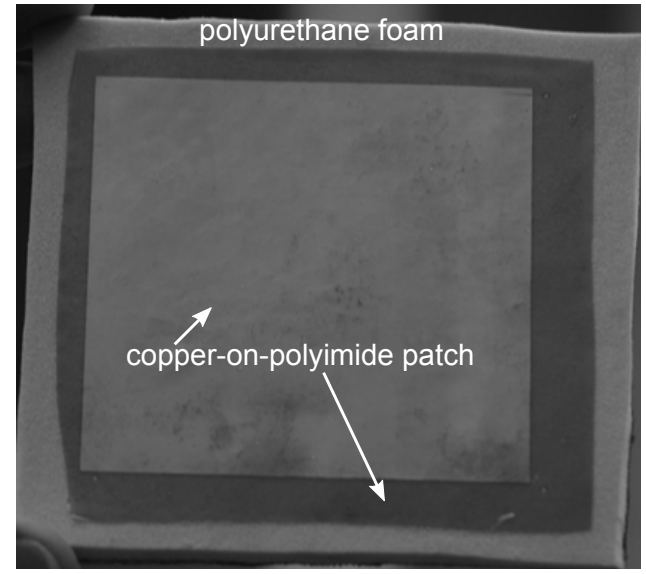

Fig. 13. Picture of the patch antenna of the active GPS antenna

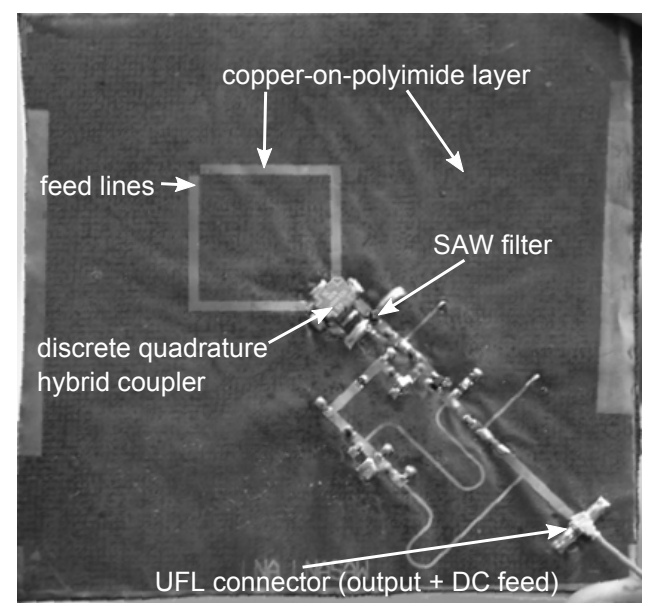

Fig. 14. Picture of the LNA of the active GPS antenna

the complete layout after each change in stub length. After optimization, the complete layout including stub waveguides was simulated to validate this approach. The optimization led to an LNA with an $18.1 \mathrm{~dB}$ gain and a noise figure of $0.475 \mathrm{~dB}$. The optimized design was fabricated, producing an active antenna measuring $10 \mathrm{~cm} \times 10 \mathrm{~cm}$ with a weight of $14 \mathrm{~g}$. Pictures of the antenna front- and backside are shown

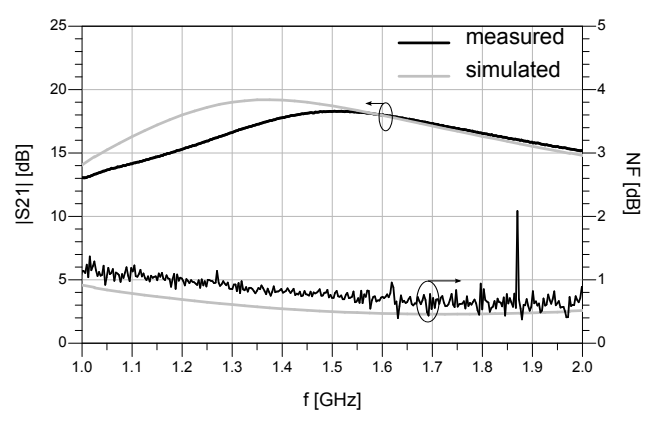

Fig. 15. LNA gain and noise performance

in Figs. 13 and 14 respectively.

\section{Design Evaluation}

Anechoic chamber measurements were carried out to evaluate the active antenna performance. Passive and active versions of the antenna were fabricated, as well as a standalone LNA. First, the LNA measurements will be discussed. Next, the active antenna measurement results are treated.

1) LNA Performance Validation: For an outline of the measurement method applied to the standalone LNA, we refer to section II-C1. The measured and simulated LNA gain and noise figure, depicted in Fig. 15, show a good agreement, the gain being about $18.1 \mathrm{~dB}$ at the GPS L1 frequency in both the simulation and the measurement, and the measured noise figure being $0.545 \mathrm{~dB}$ at the GPS L1 frequency, in good agreement with the simulated value of $0.475 \mathrm{~dB}$. The $S_{11}$ and $S_{22}$ measurements, however, differed from the simulated values, resulting in an $S_{11}$ value exceeding $-10 \mathrm{~dB}$ at the GPS L1 frequency. In the measurement setup, the LNA was interfaced with UFL connectors and cables not included in the simulations, which might explain the noted differences.Stable behaviour was observed during the measurements and the connection to an off-the-shelf GPS receiver, during which the LNA was always connected to $50 \Omega$ devices.

2) Active Antenna Performance Validation: In Figs. 16 and 17 , the gain, axial ratio and $\left|S_{11}\right|$ of the passive antenna are shown, resulting from simulations and measurements respectively. The difference between the simulated and measured results is clearly noticeable. As stated in section III-B, we did 


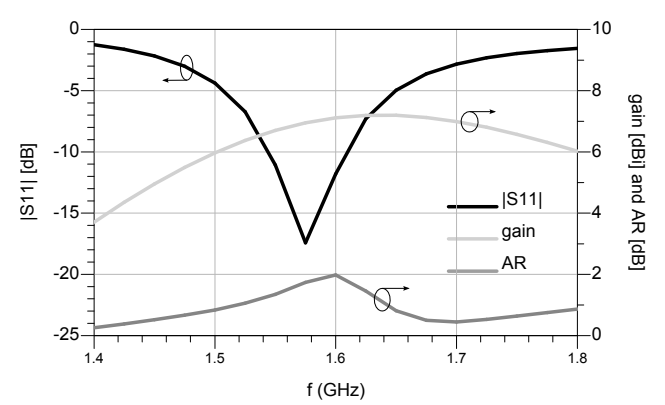

Fig. 16. Simulated passive antenna gain, axial ratio and $\left|S_{11}\right|$

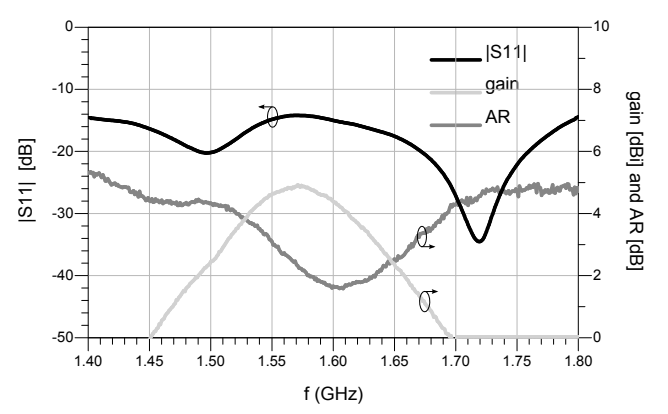

Fig. 17. Measured passive antenna gain, axial ratio and $\left|S_{11}\right|$

not include the discrete hybrid coupler in the simulations. We immediately notice its effect in the broad $50 \Omega$ match of the measured antenna. We also note differences in antenna gain and axial ratio. The measured gain at the GPS L1 frequency is about $5 \mathrm{dBi}$, compared to a simulated value of $7 \mathrm{dBi}$. The measured axial ratio at $1.575 \mathrm{GHz}$ is $2.2 \mathrm{~dB}$, which is higher than simulated, but still well below $3 \mathrm{~dB}$. Active antenna gain, axial ratio and $S_{11}$ are shown in Fig. 18. We notice an $\left|S_{11}\right|$ of $-9 \mathrm{~dB}$ at $1.575 \mathrm{GHz}$, which could be expected, taking into account the LNA measurements discussed in Section III-C1. We see a total active antenna gain of $23.6 \mathrm{~dB}$, as was to be expected from the seperate LNA/antenna measurements. The axial ratio at GPS $\mathrm{L} 1$ is about $1 \mathrm{~dB}$, which is lower than the passive antenna axial ratio. This could be explained by small differences in the antennas due to the layer alignment process. Upon connection of the active antenna with a commercial GPS-receiver, performance was noted to be better than that of an off-the-shelf rigid active antenna provided with the receiver, the flexible design locking onto a higher average number of satellites in view with higher carrier-to-noise ratios.

\section{CONCLUSION}

In this paper, two distinct active antenna design cases were presented, along with the adopted design strategies, illustrated by simulations and measurements. In Case I a good agreement between the modeled and measured results was observed. The design approach starting with a separate LNA/antenna optimization and ending with a combined LNA/antenna cooptimization proved to be very suited for the design at hand. In the second case, were the LNA and antenna were designed seperatly with $50 \Omega$ terminations, we noticed differences

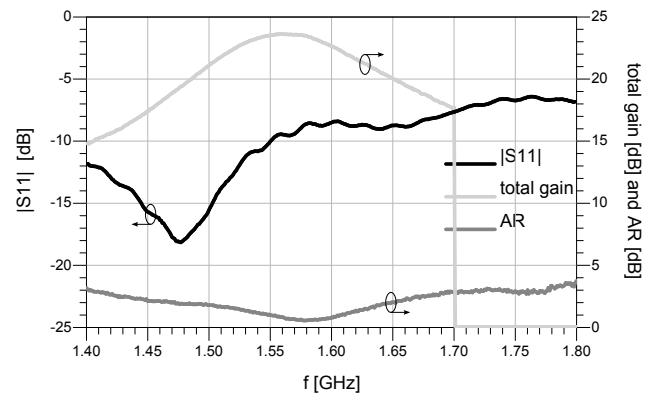

Fig. 18. Measured active antenna gain, axial ratio and $\left|S_{11}\right|$

between the simulated and measured values, which was to be expected, since the hybrid coupler was not included in the simulations. A second design step in the adopted strategy, where more elements are modeled (e.g. hybrid coupler, UFL connectors) might provide more accurate results. Nonetheless the design strategy resulted in an adequately functioning GPS antenna.

\section{REFERENCES}

[1] T. Kennedy, P. Fink, A. Chu, N. Champagne, G. Lin, and M. Khayat, "Body-worn E-Textile antennas: The good, the low-mass, and the conformal," Antennas and Propagation, IEEE Transactions on, vol. 57, no. 4, pp. 910-918, 2009.

[2] L. Vallozzi, W. Vandendriessche, H. Rogier, C. Hertleer, and M. Scarpello, "Design of a protective garment GPS antenna," Microwave and optical technology letters, vol. 51, no. 6, pp. 1504-1508, 2009.

[3] C. Hertleer, H. Rogier, L. Vallozzi, and L. Van Langenhove, "A textile antenna for off-body communication integrated into protective clothing for firefighters," Antennas and Propagation, IEEE Transactions on, vol. 57, no. 4, pp. 919-925, 2009.

[4] A. Tronquo, H. Rogier, C. Hertleer, and L. Van Langenhove, "Robust planar textile antenna for wireless body LANs operating in $2.45 \mathrm{GHz}$ ISM band," Electronics Letters, vol. 42, no. 3, pp. 142-143, 2006.

[5] C. Hertleer, A. Tronquo, H. Rogier, L. Vallozzi, and L. Van Langenhove, "Aperture-coupled patch antenna for integration into wearable textile systems," Antennas and Wireless Propagation Letters, IEEE, vol. 6, pp. 392-395, 2007.

[6] C. Hertleer, A. Tronquo, H. Rogier, and L. Van Langenhove, "The use of textile materials to design wearable microstrip patch antennas," Textile Research Journal, vol. 78, no. 8, p. 651, 2008

[7] L. Vallozzi, H. Rogier, and C. Hertleer, "Dual polarized textile patch antenna for integration into protective garments," Antennas and Wireless Propagation Letters, IEEE, vol. 7, pp. 440-443, 2008.

[8] P. Van Torre, L. Vallozzi, C. Hertleer, H. Rogier, M. Moeneclaey, and J. Verhaevert, "Dynamic link performance analysis of a rescue worker's off-body communication system using integrated textile antennas," Science, Measurement \& Technology, IET, vol. 4, no. 2, pp. 41-52, 2010.

[9] L. Vallozzi, P. Van Torre, C. Hertleer, H. Rogier, M. Moeneclaey, and J. Verhaevert, "Wireless communication for firefighters using dualpolarized textile antennas integrated in their garment," Antennas and Propagation, IEEE Transactions on, vol. 58, no. 4, pp. 1357-1368, 2010.

[10] W. Scanlon and N. Evans, "Numerical analysis of bodyworn UHF antenna systems," Electronics \& communication engineering journal, vol. 13, no. 2, pp. 53-64, 2001.

[11] P. Salonen, Y. Rahmat-Samii, and M. Kivikoski, "Wearable antennas in the vicinity of human body," in Antennas and Propagation Society International Symposium, 2004. IEEE, vol. 1. IEEE, 2004, pp. 467470.

[12] P. Salonen, F. Yang, Y. Rahmat-Samii, and M. Kivikoski, "WEBGAwearable electromagnetic band-gap antenna," in Antennas and Propagation Society International Symposium, 2004. IEEE, vol. 1. IEEE, 2004, pp. 451-454.

[13] S. Zhu and R. Langley, "Dual-band wearable textile antenna on an EBG substrate," Antennas and Propagation, IEEE Transactions on, vol. 57, no. 4, pp. 926-935, 2009. 
[14] P. Salonen and Y. Rahmat-Samii, "Textile antennas: Effects of antenna bending on input matching and impedance bandwidth," Aerospace and Electronic Systems Magazine, IEEE, vol. 22, no. 3, pp. 10-14, 2007.

[15] Q. Bai and R. Langley, "Effect of bending and crumpling on textile antennas," in 2nd IET Seminar on Antennas and Propagation for BodyCentric Wireless Communications, 2009.

[16] C. Hertleer, A. Van Laere, H. Rogier, and L. Van Langenhove, "Influence of relative humidity on textile antenna performance," Textile Research Journal, vol. 80, no. 2, p. 177, 2010.

[17] F. Declercq and H. Rogier, "Active integrated wearable textile antenna with optimized noise characteristics," Antennas and Propagation, IEEE Transactions on, vol. 58, no. 9, pp. 3050-3054, 2010.

[18] A. Dierck, T. De Keulenaer, F. Declercq, and H. Rogier, "A Wearable Active GPS Antenna For Apllication In Smart Textiles," in 32nd ESA Antenna Workshop on Antennas for Space Applications, Ghent University, Belgium, 5-8 October 2010.

[19] G. Vermeeren, H. Rogier, F. Olyslager, and D. De Zutter, "Simple low-cost planar antenna for indoor communication under the Bluetooth protocol," Electronics Letters, vol. 37, no. 19, pp. 1153-1154, 2001.
[20] F. Declercq, H. Rogier, and C. Hertleer, "Permittivity and loss tangent characterization for garment antennas based on a new matrix-pencil twoline method," Antennas and Propagation, IEEE Transactions on, vol. 56, no. 8, pp. 2548-2554, 2008.

[21] A. Ward, Application Note 1222: High Intercept Low Noise Amplifier for the $18501910 \mathrm{MHz}$ PCS Band using the Agilent ATF-54143 Enhancement Mode PHEMT.

[22] D. Pozar, Microwave engineering. Wiley-India, 2009.

[23] K. Wong. Advancements in Noise Measurement. May2008Wong.pdf. [Online]. Available: http://www.ewh.ieee.org/r6/scv/ims/archives/May2008Wong.pdf

[24] H. An, B. Nauwelaers, A. Van de Capelle, and R. Bosisio, "A novel measurement technique for amplifier-type active antennas," in Microwave Symposium Digest, 1994., IEEE MTT-S International. IEEE, 1994, pp. 1473-1476.

[25] Orcam GPS30FEVK Evaluation Kit User Manual, Orcam Systems AB, 2007. 